\title{
Study on The Effects of Self-Similar Traffic on The IEEE 802.15.4 Wireless Sensor Networks
}

\author{
Chi-Ming Wong \\ Department of Electronic Engineering \\ Jinwen University of Science and Technology \\ Taipei, Taiwan \\ gordon492@gmail.com
}

\author{
Huai-Kuei Wu \\ Department of Information Networking and System \\ Administration \\ Ling Tung University \\ Taichung, Taiwan \\ huaikuei@gmail.com
}

\begin{abstract}
A significant number of previous studies have shown, however, network traffic exhibited frequently large bursty traffic possesses self-similar properties. For the future applications of wireless sensor networks (WSNs) with large number of cluster structures, such as Internet of Things (IoT) and smart grid, the network traffic should not be assumed as conventional Poisson process. We thus employ ON/OFF traffic source with the duration of heavy-tailed distribution in one or both of the states instead of Poisson traffic to be as the asymptotically self-similar traffic for experimenting on the performance of IEEE 802.15.4 WSNs. In this paper, we will show the impact on the performance of IEEE 802.15.4 WSNs in different traffic sources such as Poisson and Pareto ON/OFF distribution by ns2 simulator. For the Pareto ON/OFF distribution traffic, we demonstrate that the packet delay and throughput appear bursty-like high value in some certain time scales, especially for the low traffic load; and the throughput will be no longer bursty-like while the traffic load increases. Intuitively, the bursty-like high delay may result in loss of some important real-time packets. For the Poisson traffic, both the throughput and packet delay appear non-bursty, especially for the high traffic load.
\end{abstract}

Keywords-wireless sensor network (WSN); IEEE 802.15.4; self-similar traffic

\section{INTRODUCTION}

The IEEE 802.15.4 [1] wireless sensor networks have emerged as a promising technique for different applications with the advance of technologies in low cost and low power micro-sensors, embedded processing, radio and wireless networking. The existing and coming applications include environmental monitoring, industrial sensing and diagnostics, healthcare, smart grid and data collecting for battlefield awareness. The topology of these applications may extend to be a complex structure. A number of studies have demonstrated that the packet traffic in both local area and wide area network exhibits noticeable bursty nature over a wide range of time scales [2][3][12][13]. This fractal-like phenomenon of packet traffic should be modelled as self-similar or long-range dependent (LRD) processes, which have significantly different theoretical properties from the conventional Poisson process.

For the performance study in wireless networks medium access control (MAC) protocols, the assumption of traffic arrival process will actually impact on the result of analysis and thus play one of a major role in the performance analysis. Most existing work of performance analysis in IEEE 802.15.4 MAC protocol focused on the saturated traffic conditions [4]-[7] where all nodes always have packets to be transmitted and unsaturated traffic conditions where packet arrives at each node according to a Poisson process with a fixed rate [8]-[10]. However, based on their traffic assumption, the performance results of analysis and simulation including the throughput and packet delay are always calculated by the run time average. The traditional run time average method basically is not easy to observe the results in short time scale accurately and may cause us to lose some important messages in certain time scales; especially for the self-similar traffic. For example, a given low packet delay that is calculated by run time average in analysis or simulation may also contain a higher value at a certain moment which may cause some problems during messages delivery in real time system such as healthcare monitoring, smart grid, environmental monitoring and industrial sensing and diagnostics. Hence, it is necessary to obtain the results by the time scales average method. In the below section III and IV, we will further show that the results of run time average and time scale average are the same in Poisson traffic assumption and are the different in self-similar traffic assumption. Therefore, in order to possess a deep understanding of the performance behaviors in the IEEE 802.15.4 networks, we use run time average (section III) and time scales average (section IV) in Poisson and self-similar process as the traffic assumption to evolve more detail of results from simulation experiments in this paper.

Capturing the heavy-tailed sojourn time behavior in the OFF and/or ON states can generate traffic that is asymptotically self-similar [14]. In this paper, we simulate ON/OFF traffic by using the Pareto distribution to provide the physical basis for the self-similarity as the packet traffic source. We will demonstrate the performance results including the throughput and packet delay in whole time average and time scales average for Poisson and Pareto ON/OFF traffic respectively. The simulation results show that the packet delay presents self-similarity and the throughput is no longer selfsimilar while traffic load increases.

In [11], the model for IEEE 802.15.4 MAC protocol with geometrically distributed burst $\mathrm{ON} / \mathrm{OFF}$ traffic has been proposed. It only uses the run time average to obtain 
performance results so that the observation in certain time scale is unable to be achieved; moreover, the traffic assumption is also not for the self-similar process. In [2], authors had established experimentation for tracing collection in IEEE 802.15.4 wireless sensor networks. They concluded that the perception of ON and OFF LRD for the packet level is actually due to interference from co-located IEEE $802.11 \mathrm{~b} / \mathrm{g}$ networks. In the current circumstances, the WiFi networks accessing almost exist everywhere. This may cause self-similar traffic in the IEEE 802.15.4 networks. Thus, it is essential to take the impact of self-similar traffic on IEEE 802.15.4 networks into account.

The rest of this paper is organized as follows. Section II describes the simulation experiments and self-similar traffic overview; section III shows the run time average method; and section IV shows the time scales average method. Finally, the conclusion is made in section $\mathrm{V}$.

\section{THE IEEE 802.15.4 WIRELESS SENSOR NETWORKS AND SELF-SIMILAR TRAFFIC OVERVIEW}

In this section, we briefly describe the IEEE 802.15.4 slotted carrier sense multiple access with collision avoidance (CSMA/CA) and summarize the properties of self-similar traffic.

\section{A. IEEE 802.15.4 Wireless Sensor Networks Overview}

In the IEEE 802.15.4 beacon-enabled mode, the coordinator broadcasts the beacon frame periodically to regulate the packet transmission. A superframe starts at the beginning of a beacon frame and ends at the beginning of the next beacon frame. The superframe consists of an active and inactive period, and the coordinator will determine their lengths by set superframe order (SO) and beacon order (BO) to be $48 x$ $2^{\mathrm{SO}}$ UnitBackoffPeriods (UBPs) and $48 \times\left(2^{\mathrm{BO}}-2^{\mathrm{SO}}\right)$ UBPs respectively, where the UBP is equal to 20 symbols while a symbol is defined as the time that four data bits are transmitted [17].

Each node transmits data to the coordinator by using the slotted CSMA/CA. In the slotted CSMA/CA algorithm, a node first delays a backoff period (BP) between 0 and $\left(2^{\mathrm{BE}}-1\right)$ slots and performs the clear channel assessment (CCA) to detect channel condition, where $\mathrm{BE}$ is a backoff exponent initiated by the value of $a M i n B E$. Node will start to transmit if two successive CCAs detect idle channel. Conversely, if any CCA detects busy channel, node should delay a BP between 0 and $\left(2^{(\mathrm{BE}+1)}-1\right)$ slots and attempt to perform CCAs again. The BE can be increased to maximum value of $a M a x B E$. The transmission fails if the number of backoff attempts exceeds the value macMacCSMABackoffs [17].

\section{B. Self-Similar Traffic Overview}

An object can be called self-similar if some of its properties are preserved with respect to scaling in space and/or time. If an object is self-similar or fractal, its parts, when magnified, resemble the shape of the whole. There are two kinds of selfsimilarity: deterministic self-similarity in which there is a strong form of recursive regularity and stochastic selfsimilarity, more general than the previous one [16].

With the idea that self-similar traffic can be studied using stochastic processes, this paragraph presents some important mathematical concepts on stochastic processes. Let $\mathrm{X}(\mathrm{t})$ be a covariance stationary stochastic process (or also second-order stationary process) with mean $\mu=\mathrm{E}[\mathrm{X}(\mathrm{t})]$ (for simplicity, in the following paragraphs, $\mu=0)$, variance $\sigma^{2}=E\left[(X(t)-\mu)^{2}\right]$ for all $\mathrm{t} \in \mathrm{Z}$ and autocovariance function $\gamma(\mathrm{k}), \mathrm{k} \geq 0$. Let $\mathrm{X}(\mathrm{m})$ be the aggregate process of $\mathrm{X}$ at aggregation level $\mathrm{m}$ defined as $X^{(m)}(i)=\frac{1}{m} \sum_{t=m(i-1)+1}^{m i} X(t)$ for $\mathrm{m}=1,2,3, \ldots$ and $\mathrm{i}>1$. Let $\gamma^{(\mathrm{m})}(\mathrm{k})$ denote the autocovariance function of $\mathrm{X}^{(\mathrm{m})}$. $\mathrm{X}(\mathrm{t})$ is exactly second-order self- similar with Hurst parameter $\mathrm{H}$ if $\gamma(\mathrm{k})=\left(\sigma^{2}\right.$ $\left.\left((\mathrm{k}+1)^{2 \mathrm{H}}-2 \mathrm{k}^{2 \mathrm{H}}+(\mathrm{k}-1)^{2 \mathrm{H}}\right)\right) / 2$.

\section{SIMULATION EXPERIMENTS AND RESULTS}

In this section, we describe the simulation experiments that are based on the ns2 simulator [15] and show the results of simulation.

\section{A. Simulation Experiments Overview}

As shown in Fig. 1, we consider a single hop IEEE 802.15.4 wireless sensor networks with star topology consisting of a coordinator (node 0 ) and 3 sensor nodes (node 1 , 2 and 3 ) in the assumption of ideal channel conditions. All sensor nodes can communicate with the coordinator by the full data rate of $250 \mathrm{kbps}$ and no capture effect is considered. We assume that the inactive period can be neglected, that is, the SO is equal to the $\mathrm{BO}$, and the packet length $\mathrm{L}$ will be fixed in 120 bytes. The traffic load in this paper is defined as $(\mathrm{N} \times \lambda \times \mathrm{L} \times 8) / 250 \mathrm{kbps}$, while $\mathrm{N}$ represents the total sensor nodes and is equal to 3 and $\lambda$ represents the mean arrival rate. The throughput and packet delay will obtain from simulations to indicate the performance. The packet delay will include the $\mathrm{MAC}$ and queuing delay. The simulation parameters in ns2 are listed in the Table 1 .

TABLE I. THE PARAMETERS FOR SIMULATION IN NS2

\begin{tabular}{|c|c|}
\hline adhocRouting & DumbAgent \\
\hline IIType & LL \\
\hline macType & Mac/802_15_4 \\
\hline ifqType & Queue/DropTail/PriQueue \\
\hline ifqLen & Antenna/OmniAntenna \\
\hline antType & Propagation/TwoRayGround \\
\hline propType & Phy/WirelessPhy/802_15_4 \\
\hline phyType & $4.80696 \mathrm{e}-07$ \\
\hline CSThresh & $4.80696 \mathrm{e}-07$ \\
\hline RXThresh & 0 \\
\hline CPThresh & $250 \mathrm{Kbps}$ \\
\hline Channel bandwidth &
\end{tabular}




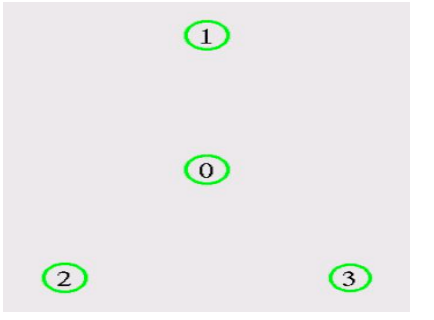

Fig. 1. Star topology in ns2 simulation

\section{B. The Run Time Average Method}

First of all, we apply the Poisson and Pareto ON/OFF distribution as the traffic source for the experiment by conventionally run time average method. The run time average method means that the results are averaged by whole simulation time. Fig. 2 and Fig. 3 show the throughput and packet delay versus traffic load in 1000 seconds simulation time. In a general point of view, obviously, the throughput of Poisson traffic source is higher than the Pareto ON/OFF traffic source whenever the traffic load exceeds in 0.5. Similarly, the average delay of Poisson traffic source will be clearly lower than the Pareto ON/OFF traffic source while the traffic load is larger than 0.5. Therefore, we may conclude that the performance of applying Poisson traffic could be almost same as the Pareto ON/OFF traffic while the traffic load is less than 0.5 in the IEEE 802.15.4 networks. Once the traffic load exceeds in 0.5 , the performance of applying Pareto ON/OFF traffic source will be inferior to the Poisson traffic source. However, for the sake of caution, we double check above conclusions by using the time scales average method in the next section.

\section{The Time Scales Average Method}

The time scales average method means that the results are averaged by the interval of time scale of whole simulation time. In this section, we demonstrate the results of throughput and packet delay versus time in time scales $100 \mathrm{~ms}, 10 \mathrm{~s}$ and $100 \mathrm{~s}$

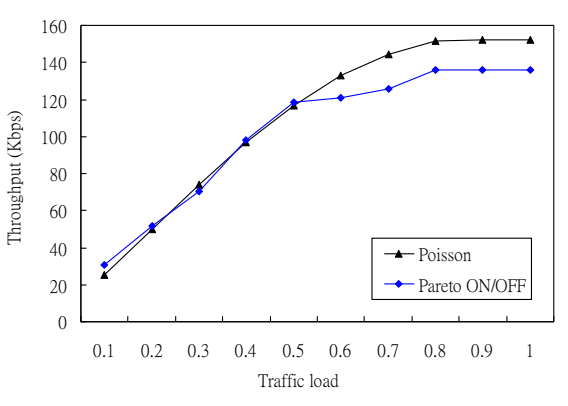

Fig. 2. Throughput vs. traffic load

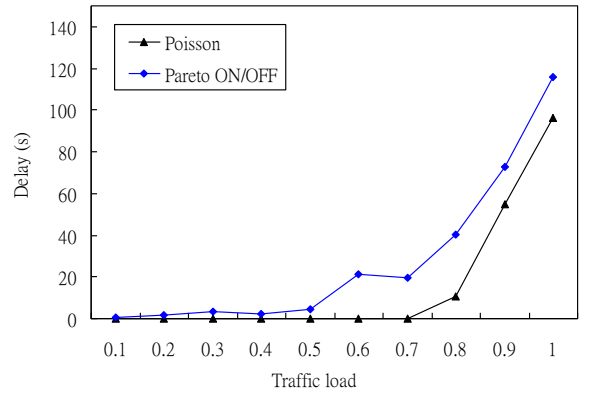

Fig. 3. Packet delay vs. traffic load

corresponding to simulation time $10 \mathrm{~s}, 1000 \mathrm{~s}$ and $10000 \mathrm{~s}$ respectively.

In Fig. 4 group, they show the throughput versus time with traffic load $0.2,0.5$ and 0.8 in time scale $100 \mathrm{~ms}$. In the light traffic load 0.2 of the Pareto ON/OFF traffic source, as shown in Fig. 4(a), clearly, it appears a bursty-like throughput. When the traffic load increases, as shown in Fig. 4(b) and Fig. 4(c), the throughput has no longer bursty and it shows the uniformity in each time scales. Fig. 4(d), Fig. 4(e) and Fig. 4(f) show the throughput in Poisson traffic source. Obviously, they appear almost in uniformity for each time scales. In Fig. 5 group, they show the packet delay versus time with traffic load 0.2, 0.5 and

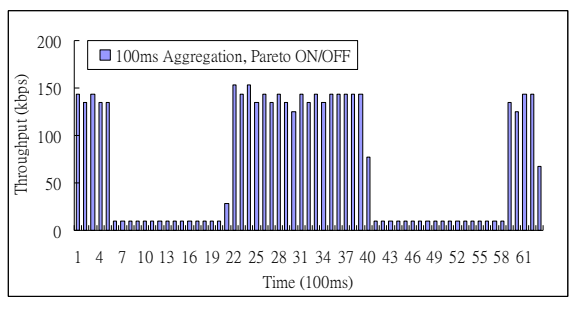

(a) Traffic load $=0.2$ in Pareto ON/OFF source

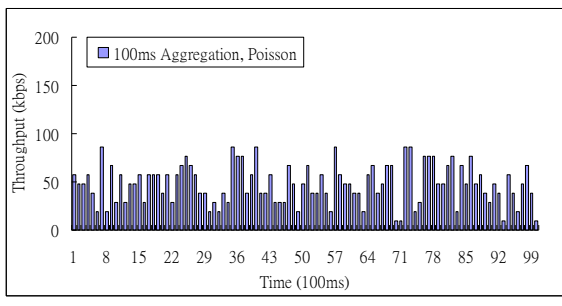

(d) Traffic load $=0.2$ in Poisson traffic source

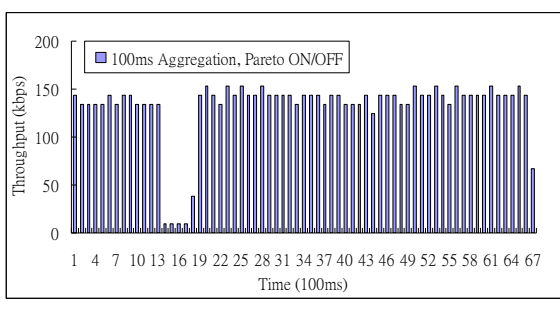

(b) Traffic load $=0.5$ in Pareto ON/OFF source

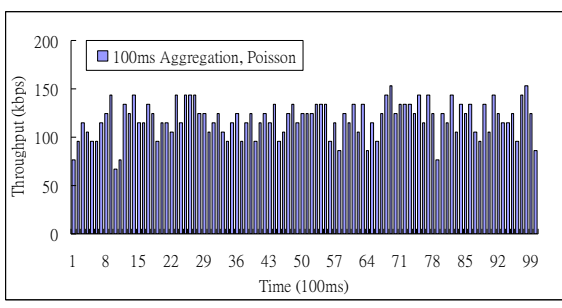

(e) Traffic load $=0.5$ in Poisson traffic source

Fig. 4. Throughput vs. time scales $100 \mathrm{~ms}$

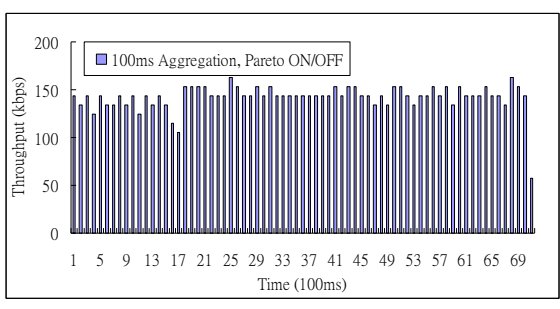

(c) Traffic load $=0.8$ in Pareto ON/OFF source

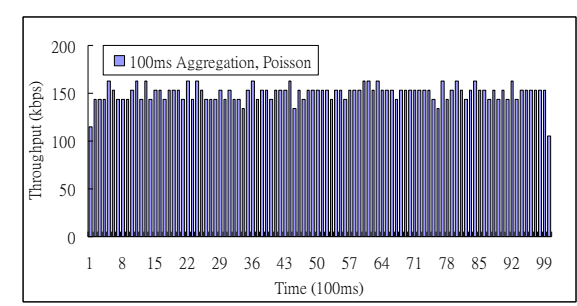

(f) Traffic load $=0.8$ in Poisson traffic source 


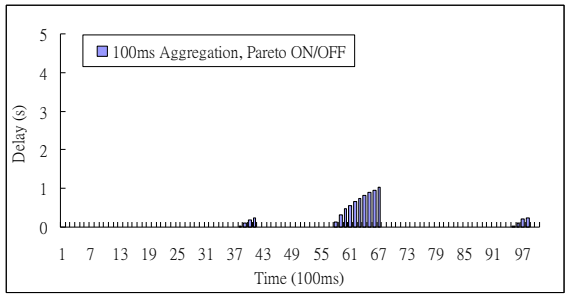

(a) Traffic load $=0.2$ in Pareto ON/OFF source

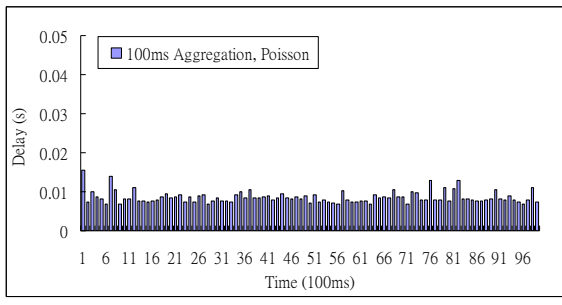

(d) Traffic load $=0.2$ in Poisson traffic source

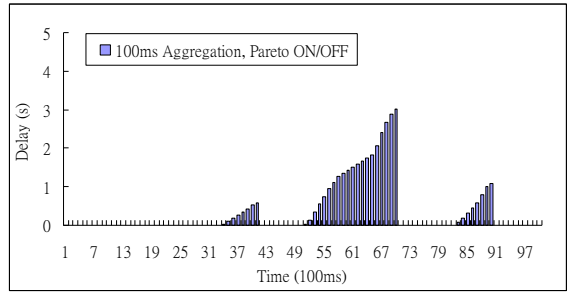

(b) Traffic load $=0.5$ in Pareto ON/OFF source

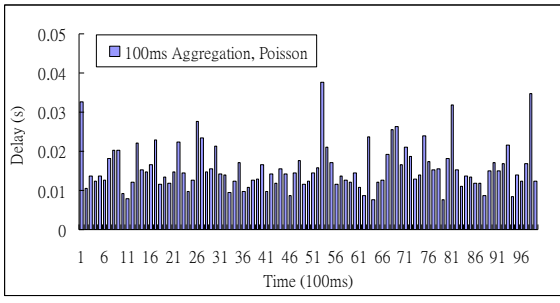

(e) Traffic load $=0.5$ in Poisson traffic source

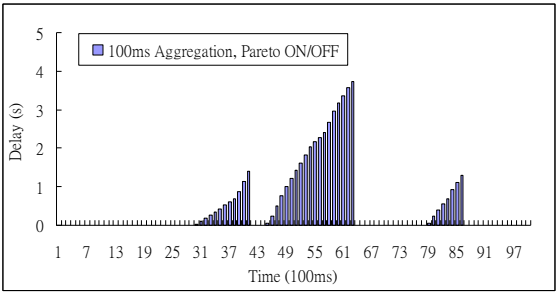

(c) Traffic load $=0.8$ in Pareto ON/OFF source

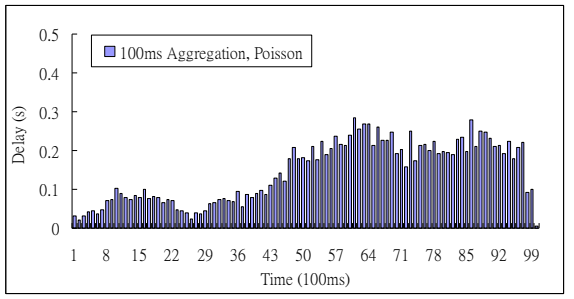

(f) Traffic load $=0.8$ in Poisson traffic source

Fig. 5. Packet delay vs. time scales $100 \mathrm{~ms}$

0.8 in time scale $100 \mathrm{~ms}$. Fig. 5(a), Fig. 5(b) and Fig. 5(c) appear bursty-like high packet delay in some certain time scales and low delay in other time zone for the Pareto ON/OFF traffic source in traffic load $0.2,0.5$ and 0.8 respectively. Compared to the Fig. 5(a), Fig. 5(b) and Fig. 5(c), the Fig. 5(d), Fig. 5(e) and Fig. 5(f) resulted from Poisson traffic source will have uniformity-like and very low packet delay. For the case of traffic load is less than 0.5 , unlike the conclusion in section B, the delay performance of Pareto ON/OFF traffic seems less than the Poisson traffic in traffic load 0.2.

The Fig. 6 and Fig. 7 group show the throughput and packet delay versus time in time scale 10 s respectively. Similarly, Fig. 6(a), Fig. 6(b) and Fig. 6(c) also show that the throughput appears bursty-like in Pareto ON/OFF traffic source with traffic load 0.2 and tends to uniformity while the traffic load increases. Fig. 6(d), Fig. 6(e) and Fig. 6(f) demonstrate that the throughput in Poisson traffic source always appears uniformity regardless of traffic load. For the delay performance in Pareto ON/OFF traffic, as shown in Fig. 7(a), Fig. 7(b) and Fig. 7(c), they also appear bursty-like high packet delay in certain time scales and very low delay in other time zone. When the Poisson traffic is applied, as shown in Fig. 7(d), Fig. 7(e) and Fig. 7(f), the delay appears uniformly low value while the traffic load is less than 0.5 and presents linearly increasing in traffic load 0.8 . In addition, Fig. 8 and Fig. 9 group show the throughput and packet delay versus time in time scale 100s. As shown in Fig. 8 (a), the traffic load is higher than or equal to 0.5 , it is quite obvious that the 0.2 traffic load of Pareto ON/OFF traffic will cause bursty-like throughput. As shown in Fig. 8(b), 8(c), 8(e) and $8(\mathrm{f})$, it presents the throughput uniformity whether the traffic source is Poisson or Pareto ON/OFF distribution. In Fig. 9(a) and Fig. 9(b), the packet delay appears bursty-like high packet delay in certain time scales when the Pareto ON/OFF

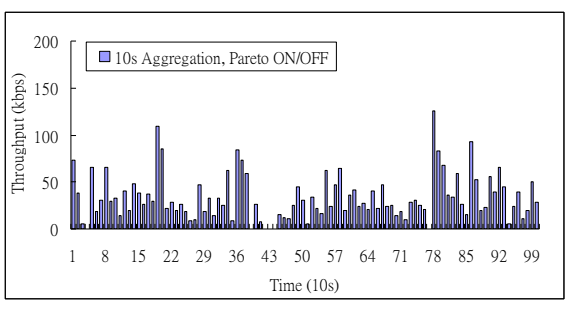

(a) Traffic load $=0.2$ in Pareto ON/OFF source

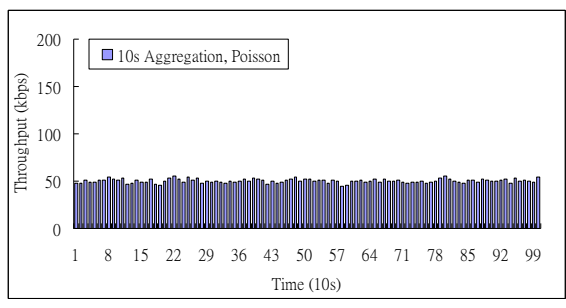

(d) Traffic load $=0.2$ in Poisson traffic source

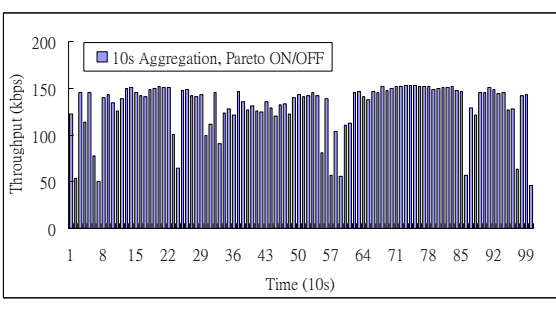

(b) Traffic load $=0.5$ in Pareto ON/OFF source

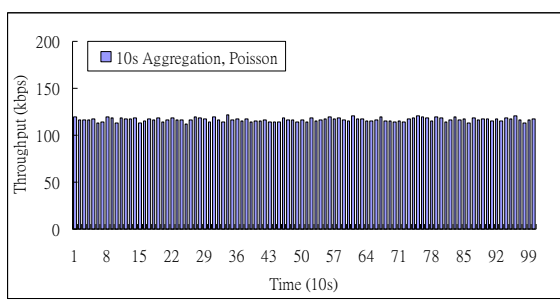

(e) Traffic load $=0.5$ in Poisson traffic source

Fig. 6. Throughput vs. time scales 10s

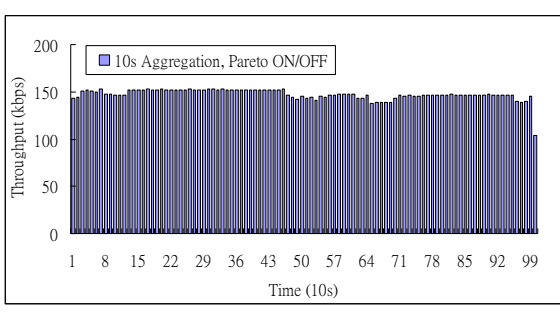

(c) Traffic load $=0.8$ in Pareto ON/OFF source

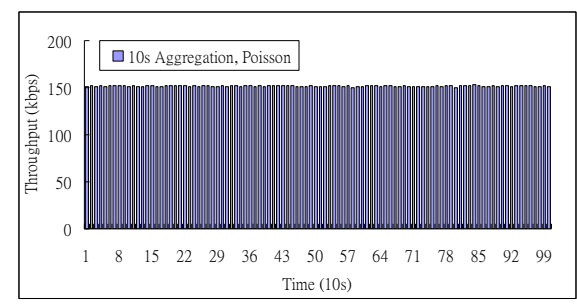

(f) Traffic load $=0.8$ in Poisson traffic source 


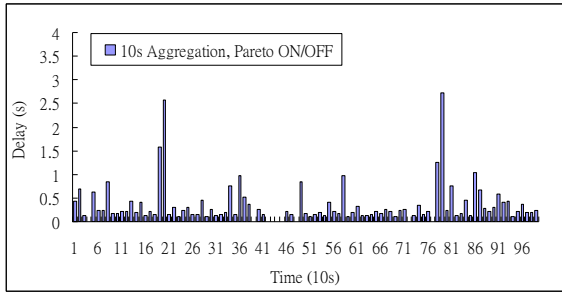

(a) Traffic load $=0.2$ in Pareto ON/OFF source

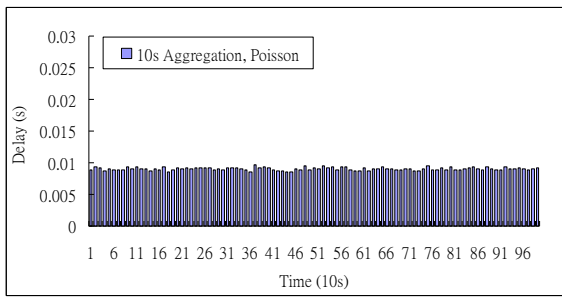

(d) Traffic load $=0.2$ in Poisson traffic source

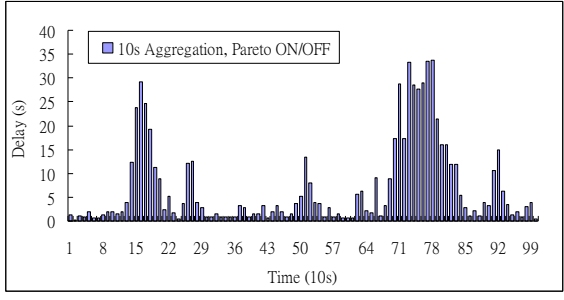

(b) Traffic load $=0.5$ in Pareto ON/OFF source

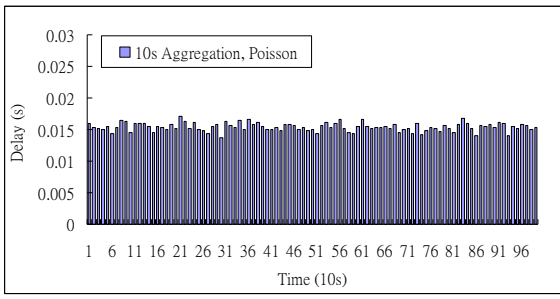

(e) Traffic load $=0.5$ in Poisson traffic source

Fig. 7. Packet delay vs. time scales 10 s

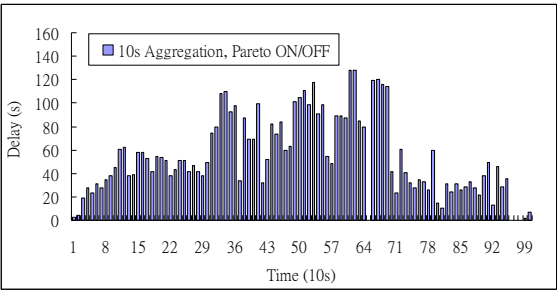

(c) Traffic load $=0.8$ in Pareto ON/OFF source

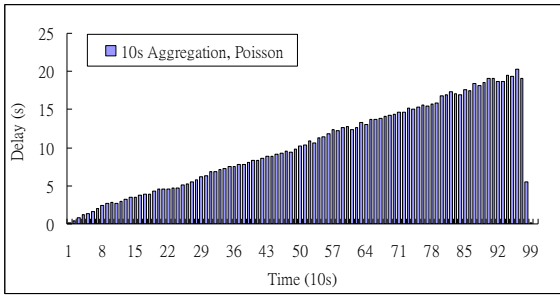

(f) Traffic load $=0.8$ in Poisson traffic source traffic is applied. In contrast, it presents more uniformity in low value when Poisson traffic is applied.

Thus, for the Poisson traffic source, the longer observing time scale, the more uniformity appears in throughput performance; and the delay is also more uniformity when the traffic load is less than 0.5 . For the Pareto ON/OFF traffic source, the longer observing time scale, the more bursty-like high packet delay in some certain time scales, especially for the traffic load below 0.5. It is different from the above conclusion that the performance of applying Poisson traffic could be almost same as the Pareto ON/OFF traffic while the traffic load is less than 0.5 in section $\mathrm{B}$ run time average method.

\section{CONCLUSION}

In this paper, we have demonstrated the performance of throughput and packet delay in IEEE 802.15.4 wireless sensor networks based on the Poisson and Pareto ON/OFF traffic. For the run time average method, the results of throughput and packet delay in Poisson and Pareto ON/OFF traffic are almost same while the traffic load is less than 0.5 . For the time scale average method, the delay performance in Pareto ON/OFF traffic appears bursty-like high value in some certain time scales, especially for the traffic load below 0.5 . The bursty-like high delay may result in loss of some important real-time packets. Hence, for some applications such as healthcare and smart grid, once the traffic source appears bursty, the unstable data reception may affect the function of the system.

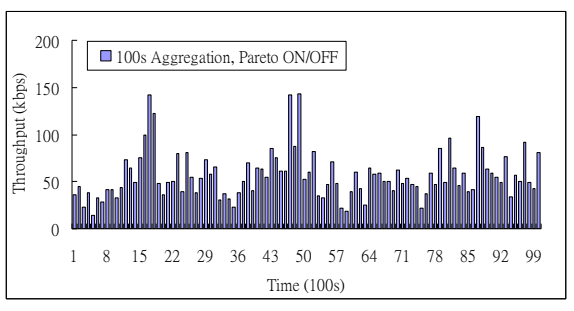

(a) Traffic load $=0.2$ in Pareto ON/OFF source

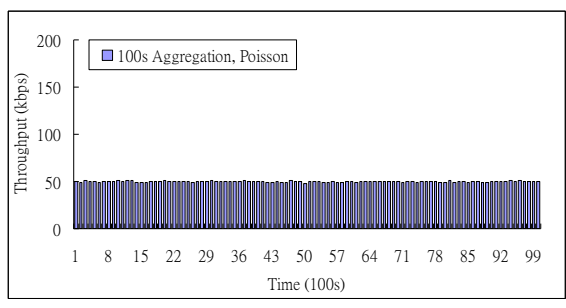

(d) Traffic load $=0.2$ in Poisson traffic source

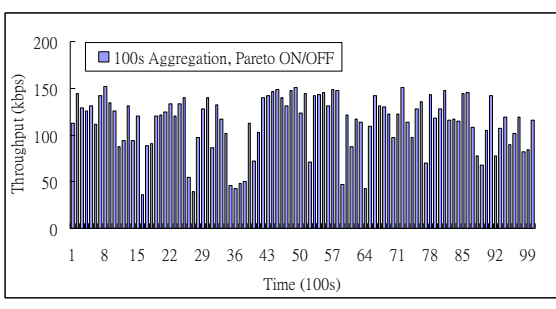

(b) Traffic load $=0.5$ in Pareto ON/OFF source

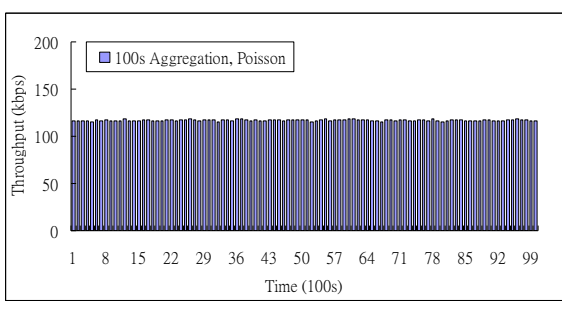

(e) Traffic load $=0.5$ in Poisson traffic source

Fig. 8. Throughput vs. time scales 100s

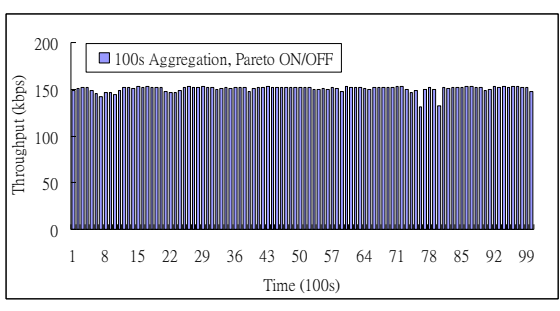

(c) Traffic load $=0.8$ in Pareto ON/OFF source

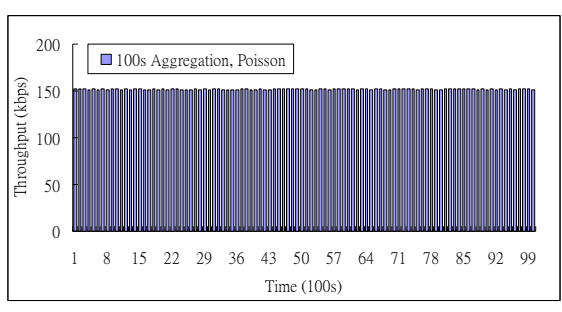

(f) Traffic load $=0.8$ in Poisson traffic source 


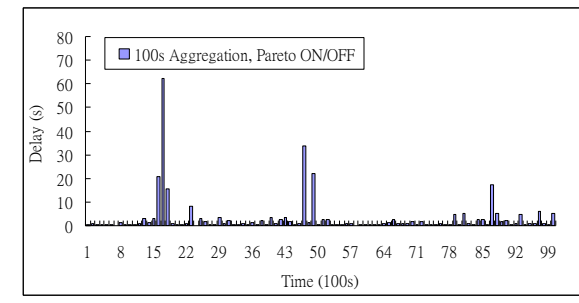

(a) Traffic load $=0.2$ in Pareto ON/OFF source

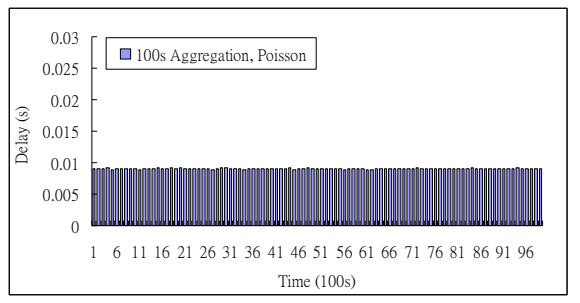

(d) Traffic load $=0.2$ in Poisson traffic source

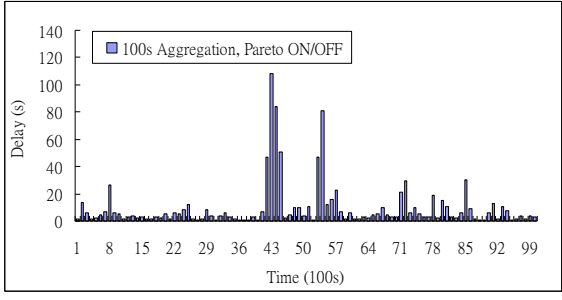

(b) Traffic load $=0.5$ in Pareto ON/OFF source

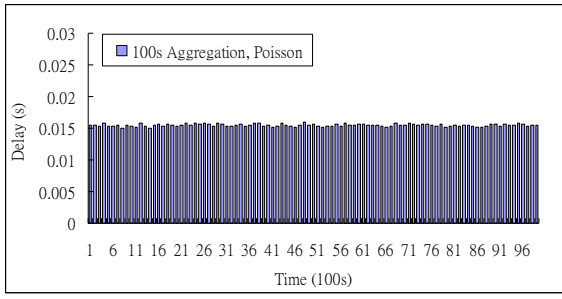

(e) Traffic load $=0.5$ in Poisson traffic source

Fig. 9. Packet delay vs. time scales 100 s

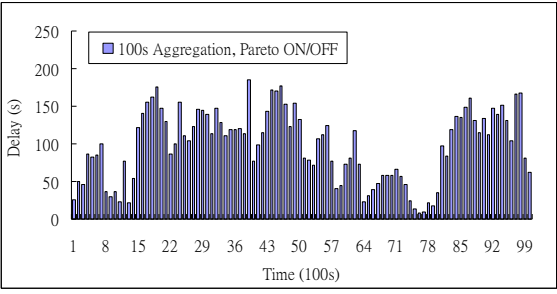

(c) Traffic load $=0.8$ in Pareto ON/OFF source

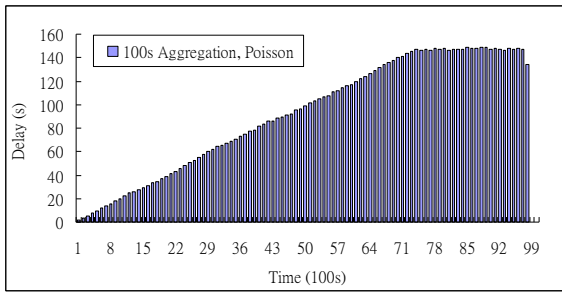

(f) Traffic load $=0.8$ in Poisson traffic source

\section{ACKNOWLEDGMENT}

This work was supported in part by the National Science Council (NSC) of Taiwan under Grant No.: NSC 102-2221-E228-001.

\section{REFERENCES}

[1] IEEE 802.15.4 Working Group, "Standard for Part 15.4: Wireless Medium Access Control Layer (MAC) and Physical Layer (PHY) Specifications for Low Rate Wireless Personal Area Networks (LRWPANs)," IEEE Std. 802.15.4, Oct. 2003.

[2] M. U. Ilyas and H. Radha, "Long range dependence of IEEE 802.15.4 wireless channels," in Proc. IEEE International Conference on Communications, pp. 4261-4265, May 2008.

[3] S. A. Stoev, G. Michailidis, and M. S. Taqqu, "Estimating heavy-tail exponents through max self-similarity," IEEE Transations on Iformation Theory, vol. 57, no. 3, pp. 1615-1636, Mar. 2011.

[4] B. J. Kwak, N. O. Song and L. E. Miller, "Performance Analysis of Exponential Backoff," IEEE/ACM Transactions on Networking, vol. 13, no. 2, pp. 343-355, Apr. 2005.

[5] T. Park, T. Kim, J. Choi, S. Choi, and W. Kwon, "Throughput and Energy Consumption Analysis of IEEE 802.15.4 Slotted CSMA/CA," IET Electronics Letters, vol. 41, no. 18, pp. 1017-1019, Sept. 2005.

[6] S. Pollin, M. Ergen, S. Ergen, B. Bougard, L. V. der Pierre, F. Catthoor, I. Moerman, A. Bahai, and P. Varaiya, "Performance Analysis of Slotted Carrier Sense IEEE 802.15.4 Medium Access Layer," IEEE Transactions on Wireless Communications, vol. 7, no. 9, pp. 3359-3371, Sept. 2008.

[7] M. D. Francesco, G. Anastasi, M. Conti, S. K. Das and V. Neri, "Reliability and Energy-Efficiency in IEEE 802.15.4/ZigBee Sensor Networks: An Adaptive and Cross-Layer Approach," IEEE Journal on Selected Areas in Communications, vol. 29, no. 8, pp. 1508-478, Sept. 2011 .
[8] I. Ramachandran, A. K. Das, and S. Roy, "Analysis of the Contention Access Period of IEEE 802.15.4 MAC," ACM Transactions on Sensor Networks, vol. 3, pp. 1-29, Mar. 2007.

[9] C. Y. Jung, H. Y. Hwang, D. K. Sung and G. U. Hwang, "Enhanced Markov Chain Model and Throughput analysis of the Slotted CSMA/CA for IEEE 802.15.4 Under Unsaturated Traffic Conditions," IEEE Transactions on Vehicular Technology, vol. 58, no. 1, pp. 473-478, Jan. 2009.

[10] P. D. Marco, P. Park, C. Fischione and K. H. Johansson, "Analytical Modeling of Multi-hop IEEE 802.15.4 Networks," IEEE Transactions on Vehicular Technology, vol. 61, no. 7, pp. 3191-3208, Sept. 2012.

[11] J. Gao, J. Hu and G. Min, "Performance Modelling of IEEE 802.15.4 MAC in LR-WPAN with Bursty ON-OFF Traffic," in Proc. IEEE $9^{\text {th }}$ International Conference on Computer and Information Technology, pp. 58-62, Mar. 2009.

[12] A. Erramilli, O. Narayan, and W. Willinger, "Experimental Queuing Analysis with Long-Range Dependent Packet Traffic," IEEE/ACM Transactions Networking, vol. 4, pp. 209-223, Apr. 1996.

[13] V. Paxon and S. Floyd, "Wide Area Traffic: The Failure of Poisson Modeling," IEEE/ACM Transactions Networking, vol. 3, pp. 226-244, June 1995.

[14] P. Pruthi, "Heavy-Tailed ON/OFF Source Behavior and Self-similar Traffic," in Proc. IEEE/ACM Transactions Networking, vol. 3, pp. 445-450, June 1995.

[15] http://www.isi.edu/nsnam/ns/

[16] M. Marco, A. Cenedese, S. Vitturi and T. Federico, "Traffic Models for Data Networks and Energy Efficient Control Techniques," Master thesis in Department of Infomation Engineering Master of Science in Automation Engineering, 2014.

[17] C. M. Wong, C. F. Chang and B. H. Lee, "A Simple Time Shift Scheme for Beacon Broadcasting Based on Cluster-Tree IEEE 802.15.4 LowRate WPANs," Wireless Pers Commun, vol. 72, no. 4, pp. 2837-2848, Oct. 2013. 